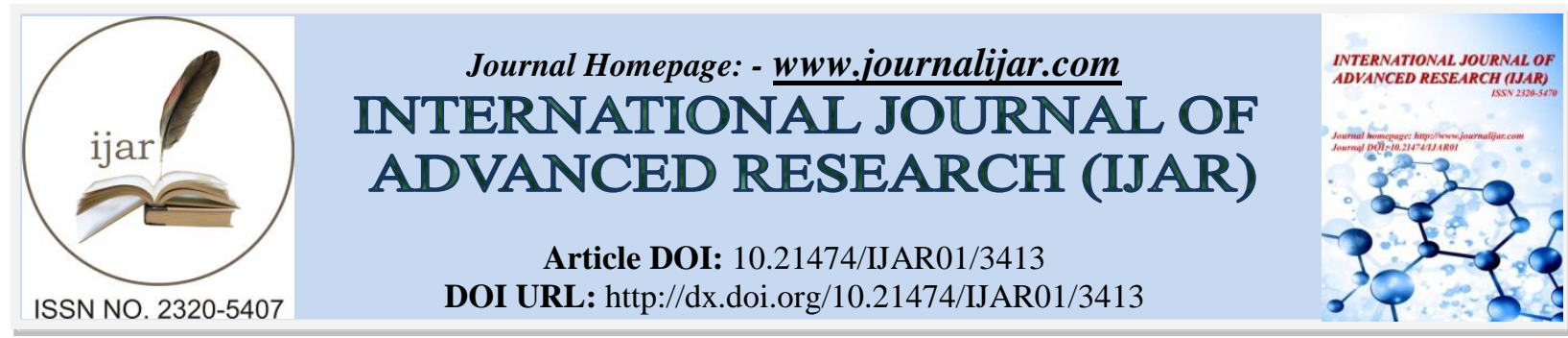

RESEARCH ARTICLE

\title{
HISTOLOGICAL EVALUATION OF RESPONSE TO DIRECT PULP CAPPING WITH PROPOLIS: EXPERIMENTAL STUDY IN RABBIT.
}

\section{Asmaa M Gamal ${ }^{1}$, Nagwa M Khattab ${ }^{2}$, Tamer A Fouda ${ }^{3}$ and Safaa M Tohamy ${ }^{4}$.}

1. Dentist in Faculty of Dentistry Hospital.

2. Professor of Pediatric and Community Dentistry and Vice Dean for education and student Affairs, Faculty of Dentistry, Minia University.

3. Lecturer of Pediatric and Community Dentistry, Faculty' of Dentistry', Minia University.

4. Lecturer of Oral Pathology, Faculty of Dentistry, Minia University.

\section{Manuscript Info}

\section{Manuscript History}

Received: 12 December 2016

Final Accepted: 15 January 2017

Published: February 2017

Key words:-

propolis, calcium hydroxide, pulp capping, rabbits.

\begin{abstract}
Herbals have been used for centuries to prevent and control disease. Herbal extracts are effective because they interact with specific chemical receptors within the body and are in a pharmacodynamic sense than drugs themselves so usage of herbal extract averte many patients from many side effects that generally come with traditional medicines. Propolis gained a popularity in the field of Dentistry because of its antimicrobial, anti-inflammatory, healing, anesthetic and cariostatic properties. The aim of the current study was to evaluate the histological response of a healthy rabbit pulp to direct pulp capping with propolis compared to calcium hydroxide. Twelve male rabbits were selected, their dental pulps were intentionally exposed by using low speed round bur on labial surfaces of permanent central incisors. Split mouth technique was used for applying the capping material to control and experimental groups. Each group was subdivided into three subgroups, four rabbits for each, where rabbits were sacrificed after 1 , 2 and 4 weeks from capping time respectively. Teeth were dissected after animal scarification and prepared for histopathologic and histochemical evaluation using Hematoxylin_ Eosin (HE) and Trichrome stains. The results showed that $25 \%, 50 \%$ and $25 \%$ of dental pulps capped with calcium hydroxide showed mild, moderate and severe inflammatory response respectively, while in propolis group $75 \%$ and $25 \%$ showed slight to moderate inflammatory response respectively. As regard hard tissue formation in response to capping materials, in calcium hydroxide group half of the cases showed moderate deposition and other half showed marked hard tissue deposited at fourth week of follow up period. While in propolis group there was a marked deposition in $75 \%$ of cases and moderate deposition in $25 \%$ in the other. Conclusion propolis proved to have less intense inflammatory response and better quality dentin bridge formation.
\end{abstract}

Copy Right, IJAR, 2017,. All rights reserved. 


\section{Introduction:-}

Protection of the dentin-pulp complex consists of the application of one or more layers of specific material between the restorative material and dental tissue to avoid additional challenge to the pulp tissue caused by operative procedures, toxicity of restorative materials and bacteria penetration due to microleakage. Protection of the dentin pulp complex has also the function to recover pulp vitality (Briso et al., 2006).

Several materials have been suggested for capping dental pulps, however calcium hydroxide is the gold standard for pulp capping (Accornite et al., 2005), the hydroxyl group is considered to be the most important component of $\mathrm{Ca}$ $(\mathrm{OH})_{2}$ as it provides an alkaline environment, which encourages repair and active calcification. The alkaline $\mathrm{pH}$ induced not only neutralizes lactic acid from osteoclasts, thus preventing dissolution of the mineral components of dentine, but could also activate alkaline phosphatase that plays an important role in hard-tissue formation. The $\mathrm{pH}$ necessary for the activation of this enzyme varies from 8.6 to 10.3. Alkaline phosphatase separate phosphoric esters, freeing phosphate ions, which then react with calcium ions to form a precipitate, calcium phosphate, in the organic matrix. This precipitate is the molecular unit of hydroxyl apatite which is believed to be intimately related to the process of mineralization (Estrela et al., 1999).

Calcium hydroxide initially bactericidal then bacteriostatic, promotes healing and repair, stimulates fibroblasts, neutralizes low $\mathrm{pH}$ of acids, stops internal resorption, inexpensive and easy to use, but also has some disadvantages as does not exclusively stimulate dentinogenesis, associated with primary tooth resorption, may dissolve after one year with cavosurface dissolution, may degrade during acid etching, degrades upon tooth flexure, marginal failure with amalgam condensation and does not adhere to dentin or resin restoration (Mustafa et al., 2012).

Mineral Trioxide Aggregate (MTA) is similar to calcium hydroxide, including its antibacterial and biocompatibility properties, high $\mathrm{pH}$, radiopacity and its ability to aid in the release of bioactive dentin matrix proteins. There are some differences between MTA and calcium hydroxide, first, MTA comes in two colors, white and grey. The grey version is due to the addition of iron. Another significant difference is the fact that MTA provides some seal to tooth structure (Luketic et al., 2008).

However there are several disadvantages with MTA, it has shown high solubility, demonstrating $24 \%$ loss after 78 days of storage in water, and prolonged setting time of approximately 2 hours and 45 minutes so pulp capping with MTA should be done in a two-step procedure, placing a temporary restoration to allow the MTA to set before placing the permanent restoration. One gram of MTA powder costs approximately the same as 24 grams of calcium hydroxide base/catalyst paste, making MTA much more cost (Fridland and Rosado, 2005).

Glass ionomer has the ability to bond chemically with tooth structure, so it can prevent the diffusion of potentially toxic materials through dentin to the pulp. Glass ionomer also provides an excellent bacterial seal and shows good biocompatibility when used in close approximation but not in direct contact with the pulp (Costa et al., 2003).

Glass ionomer was also tested as dental pulp capping material. Nascimento et al., (2000) evaluated the response of human pulps after capping with calcium hydroxide and resin modified glass ionomer. It was found that resin modified glass ionomer caused a moderate to intense inflammatory reaction with a large area of necrotic zone and lack of dentin bridge formation, while calcium hydroxide allowed pulp repair and complete dentin bridging around the pulp exposure. These results suggested that resin modified glass ionomer is not suitable to be used in direct pulp capping for mechanically exposed human pulps.

Propolis has antibacterial, antifungal, antioxidant and anti-inflammatory properties (Koru et al., 2007), these biological properties are related to its chemical composition of flavonoids, phenolics, and aromatic compounds (Park et al., 1998).

The method of extraction and type of solvent can change the chemical composition of propolis extract. Commercial products such as tablets, capsules, ampoules, and syrups are prepared with ethanolic extract of propolis. Methanol is only used for research purposes (Xu et al., 2009).

Therefore, the aim of this study was to evaluate the histological response of a healthy rabbit pulp to direct pulp capping with propolis compared to calcium hydroxide. 


\section{Materials and method:-}

Twelve male rabbits weighed $2.5 \mathrm{Kg}$ were selected for this study. Pulp capping procedure were performed, in which two opposing quadrant in each rabbit were assigned for each medicament (split mouth technique). Pulps were capped ether with propolis (control group) or calcium hydroxide (experimental group).

\section{Ethical regulation:-}

The research was approved by the research ethics committee, Faculty of Dentistry, Minia University.

\section{Experimental procedures:-}

Animals were anesthetized by intra-muscular injection in the quadriceps femora muscle using $3.3 \mathrm{~cm}$ of Xyla-ject solution, and then the working field was disinfected by $2 \%$ cholorohexidine solution and dried by cotton rolls. Dental pulps were intentionally exposed by using low speed round bur and coolant on labial surfaces of permanent central incisors. One central incisor was capped with calcium hydroxide and the other with propolis.

\section{Capping materials:-}

1- Calcium hydroxid (Dycal, Dentsply).

2- Propolis (Imtenan): the powder was mixed with $70 \%$ ethyl alcohol to a thick consistency on a paper pad with the aid of plastic spatula, the mix was placed on the exposure site by means of small ball burnisher and was allowed to set (setting time 3 min.)

Cavities were sealed with glass ionomer cement (Riva) after setting of capping materials.

\section{Animal care:-}

After completion of dental procedure, the animals were taken care of according to the protocol of Canadian Council on Animal Care and in coherence with the Three Rs (replacement, reduction, reinforcement) of animal ethics (Fenwick et al., 2011).

\section{Animal scarification:-}

Four rabbits in each group were sacrificed at 1,2, 4 weeks respectively. Then the upper jaw was removed and the capped teeth were dissected.

Histological evaluation (Passcoe and Gatehouse, 1986):

* Fixation of the tissue: The specimens were put immediately in fixative10\% formalin for $48-72$ hours.

* Washing: After adequate fixation in $10 \%$ formalin solution, the specimens were washed under running tap water overnight to remove the excess of the fixative.

* Decalification :Decalcifications of the specimens were carried out using $20 \%$ formic acid buffered with sodium citrate for 10 weeks.

* Dehydration: Water was removed from the tissue gradually by putting it in ascending grades of Ethyl alcohol; 50\%, 70\%, and 90\% then in absolute alcohol.

* Clearing: Since paraffin and alcohol are not miscible, the tissue was put in xylene (clearing agent) which is miscible with both alcohol, and paraffin. It also made the tissue translucent.

* Infiltration and embedding :When xylene was completely replaced the alcohol in the tissue, the specimens became clear, they were embedded in dish filled with melted paraffin then removed from the dishes with a warm forceps and placed in the a box of melted hard paraffin, the bottom of which was the surface of cutting.

* Cutting: The paraffin embedded specimens were serially cut with microtone in a buccolingual plane parallel to the tooth vertical axis through the cavity preparation and the pulp into sections of 5 microns thickness showing the deepest part of the cavity and the underlying pulp .

* Mounting: A short length of paraffin ribbon was floated in a pan of warm water (about $20^{\circ} \mathrm{C}$ ). The prepared slide was slipped under the ribbon and then lifted from the water with the ribbon, which contained the tissue sections arranged on its upper surface. The slide was placed on a constant temperature drying table which was regulated to about $37-42^{\circ} \mathrm{C}$, so that the sections adhered to the slide. The slide was then allowed to dry on this table.

The staining technique:-

A) Haematoxylin and eosin staining: to evaluate inflammatory response according to the following scores:

B) Trichrome stain :to evaluate and score tissue fibrosis 
Statistical method: Data entry and analysis were done with I.B.M. compatible computer using the software SPSS for windows version 13. The significance level was set at $\mathrm{P}<0.05$

Table 1:- Scoring system of inflammatory cell response.

\begin{tabular}{|l|l|}
\hline Characterization & Grade \\
\hline Absence of inflammation or mild inflammatory response limited to the capping site. & 0 \\
\hline $\begin{array}{l}\text { Slight to moderate inflammation below the capping site but limited to the coronal portion of the } \\
\text { radicular pulp. }\end{array}$ & 1 \\
\hline $\begin{array}{l}\text { Moderate inflammation evident below the capping site and extended to the middle one third of the } \\
\text { pulp. }\end{array}$ & 2 \\
\hline Severe inflammation affecting the whole pulp (including partial necrosis ) & 3 \\
\hline Pulp necrosis & 4 \\
\hline
\end{tabular}

Table 2:- Scoring system of tissue fibrosis

\begin{tabular}{|l|l|}
\hline Characterization & Grade \\
\hline Normal collagen fibers distribution & 0 \\
\hline Mild fibrosis & 1 \\
\hline Moderate fibrosis & 2 \\
\hline Sever fibrosis & 3 \\
\hline
\end{tabular}

Table 3:- Scoring system of hard tissue formation; dentin bridge (Parolia, et al 2010)

\begin{tabular}{|l|l|}
\hline \multicolumn{1}{|c|}{ Characterization } & Grade \\
\hline Absent & 0 \\
\hline Mild hard tissue deposition beneath the exposed area or partially formed hard tissue & 1 \\
\hline Moderate hard tissue deposition beneath the exposed area & 2 \\
\hline Heavy hard tissue deposition beneath the exposed area & 3 \\
\hline
\end{tabular}

\section{Results:-}

I- Histopathological evaluation of specimens:

1-Calcium hydroxide: (Control group)

1. a - After one week of application of its application:

The initial reaction of the pulp to the capping material in most of specimens was a superficial layer of necrosis just beneath the material. This necrosis was followed by a weak inflammatory reaction [grade (0)] in 25\% of specimens, which is characterized by the presence of dilated blood vessels and few inflammatory cells, and slight inflammation limited to coronal portion beneath the capped site grade (1) inflammatory cell responsein75\% of specimens.

1. b- After two weeks of application:-

$25 \%$ of specimens showed grade (1) inflammatory response, $25 \%$ had moderate inflammation extended to the middle third of the pulp; grade (2) inflammatory response and the other $50 \%$ showed sever inflammation extended along the pulp tissue with partial necrosis; grade (3) inflammatory response.

1. c- After four weeks of application:-

$25 \%, 50 \%$ and $25 \%$ of specimens showed grade 1,2 and 3 inflammatory responses respectively.

2- Propolis group: (Experimental group)

2. a- After one week of application:-

There was a mild inflammatory reaction grade (0) inflammatory cell response in $75 \%$ of specimens, and the other $25 \%$ showed grade (2) inflammatory cell response.

2. b- After two weeks of application:-

There were $50 \%$ of specimens showed grade (1) and the other $50 \%$ had grade (2) inflammatory cell response.

2. c- After four weeks of application:-

Grade (1) and grade (2) inflammatory tissue response respectively were evident in $75 \%$ and $25 \%$ of specimens respectively.

Histochemical evaluation of specimens:-

1- Calcium hydroxide: (Control group)

1.a) After one week of application. 
All pulps (100\% of specimens) had no fibrosis grade (0) tissue fibrosis.

1.b) After two weeks of application:

$50 \%$ of specimens showed mild fibrosis grade (1), while $25 \%$ had moderate fibrosis grade (2) and the other $25 \%$ showed severe fibrosis grade (3) tissue fibrosis.

1.c) After four weeks of application of capping material:

$75 \%$ of specimens showed grade (1) and $25 \%$ of specimens had grade (2) tissue fibrosis.

2- Propolis (experimental group):

2.a) After one week of application :

$100 \%$ of specimens had grade (0) tissue fibrosis.

2.b) After two weeks of application:

Half of the specimens showed grade (1) tissue fibrosis and the other half exihibited grade (2) tissue fibrosis.

1.c) After four weeks of application:

$50 \%$ of specimens showed grade (1) and the other $50 \%$ grade (0).

Evaluation of hard tissue formation (dentin bridge):

1-Calcium hydroxide (control group):

1.a) After one week of application.

$25 \%$ of specimens showed a thin layer of partially calcified dentin matrix, grade (1) hard tissue formation, just beneath a superficial layer of necrosis formed below the applied material and the other $75 \%$ had no evidence of hard tissue formation, grade (0).

1.b) After two weeks of application:

$75 \%$ of specimens showed grade (1) and $25 \%$ had moderate hard tissue deposition beneath the capping material, grade (2).

1.c) After four weeks of application:

$50 \%$ of specimens showed grade (2) and 50\% revealed heavy hard tissue deposition beneath the capping site, grade (3).

2- Proplois group (experimental group)

2.a) After one week of application.

Thin layer of partially calcified dentin matrix, grade (1) was seen in only $50 \%$ of specimens, other specimens showed no evidence of hard tissue formation.

2.b) After two weeks of application.

$50 \%$ of specimens showed grade (1), while the other $50 \%$ had grade (2).

2.c) After four weeks of application.

$25 \%$ of specimens showed grade (2) and $75 \%$ showed grade (3).

\section{Discussion:-}

The clinical criterion is inadequate for evaluation of the long-term prognosis for teeth treated by pulp capping. It is impossible to clinically diagnose teeth in which healing is complicated by inflammation. Therefore, a critical evaluation of pulp capping results can only be made histologically (Woehrlen, 1997).

Many studies have indicated that calcium hydroxide compounds are the gold standard for pulp capping in human teeth, despite its limitations (Accornite et al., 2005), propolis have antibacterial, antifungal, antiviral, antioxidant and anti-inflammatory proprieties, Therefore the current study aimed to compare one of natural product "propolis" to gold standard "calcium hydroxide" as pulp capping of rabbit teeth.

The animal model selected in the present study was rabbits because their pulp tissues are comparable with that of human (Belduz et al., 2010), using split mouth technique so that both medicaments tested in the same animal in alternate sides of the mouth.

The follow up period was short term extended only 4 weeks this because rabbit teeth grow or erupt continuously, these growth or eruption is held in balance by dental abrasion from chewing a diet high in fiber (Konigswald and Golenishev, 1979). Several previous studies used comparable follow up periods (Haddad et al., 2003 and Sabir et al., 2005). 
Results of the current study revealed that, specimens capped with propolis exhibited less inflammatory reaction compared to calcium hydroxide at all follow up periods. This finding goes in accordance with Sabir et al, (2005), Parolia et al, (2010), Ozorio et al, (2012), Ahangari et al., (2012) who found that propolis delay the inflammatory response. The delay of inflammatory response with propolis could be related to its anti-inflammatory property. Flavonoids and caffeic acid present in propolis are known to play an important role in reducing the inflammatory response by inhibiting the lipoxygenase pathway of arachidonic acid. Also, propolis as an antimicrobial agent could break down bacterial cell wall, cytoplasm and prevent bacterial cell division (Khayyal et al., 1993).

On the other hand study of Esmeraldo et al., (2013) found that green propolis extract produced intense inflammatory infiltrate and necrosis in root canal pulp tissue after 24, 72 hours and 7 days compared to calcium hydroxide. Difference may be attributed to difference in the studied animal, the current study was on rabbits while study was on rat, also within the second study, teeth were protected with a fragment of absorbent paper soaked with the solution so this inflammation may be stemmed from a reaction to the foreign body (absorbent paper) rather than propolis itself.

As regard to fibrosis, after one week of capping procedure, both groups did not show any signs of fibrosis [grade (0)], while after two weeks of capping procedure in calcium hydroxide group there were varies degree of tissue fibrosis $50 \%, 25 \%$ and $25 \%$ of specimens showed grades (1), (2) and (3) tissue fibrosis respectively, compared to $50 \%$ grade (1) and 50\% grade (2) tissue fibrosis in propolis group. After four weeks of application, $75 \%$ of specimens in calcium hydroxide group showed grade (1) fibrosis and the other $25 \%$ showed grade (2) fibrosis , while in propolis group half of specimens had grade $(0)$ and the other half had grade $(1$, denoting a better recovery in propolis group.

When hard tissue formation was evaluated, the results supported by previous study conducted by Paloria et al., (2010) who found percentage of specimens exhibited hard tissue bridges after 15 days are equal in calcium hydroxide and propolis group, while after 45 days $100 \%$ of specimens capped with propolis showed dentine bridge formation compared to $83 \%$ of specimens capped with calcium hydroxide. The dentin bridge formed in response to propolis was better in quality than calcium hydroxide, it was thicker and continuous. The same finding was reported by Ahangari et al., (2012) who stated that propolis not only stops inflammatory reaction, infection and pulp necrosis but also induce formation of high quality tubular dentin through stimulation of stem cells.

Dentin formation following pulp capping is known to involve differentiation of odontoblast-like cells that form reparative dentin and biosynthetic activity by surrounding primary odontoblasts. Both phenomena require interaction between extracellular matrix molecules and growth factors such as transforming growth factor (TGF)- $\beta 1$, a growth known to be important for odontoblasts-like cell differentiation (Tziafas et al., 2000). Indeed, propolis is capable of stimulating the production of (TGF)- $\beta 1$ (Ansorge et al., 2003).and synthesis of collagen by dental pulp cells (Scheller et al., 1978).

Therefore, within the limitation of the current study propolis showed less intense inflammatory reaction and better dentin bridge formation compared to calcium hydroxide, suggesting its use as a possible alternative to calcium hydroxide.

\section{Conclusion:-}

Propolis was superior to calcium hydroxide in terms of less intense inflammatory response and better quality dentin bridge formation.

\section{Recommendations:-}

1-Propolis can be suggested as a possible alternative to calcium hydroxide as pulp capping material.

2- Further studies are required to

a) Evaluate an alcohol free Propolis mix.

b) Compare Propolis with other pulp capping materials.

c) Evaluate ability of Propolis for hard tissue formation (dentinogenesis) in vivo. 


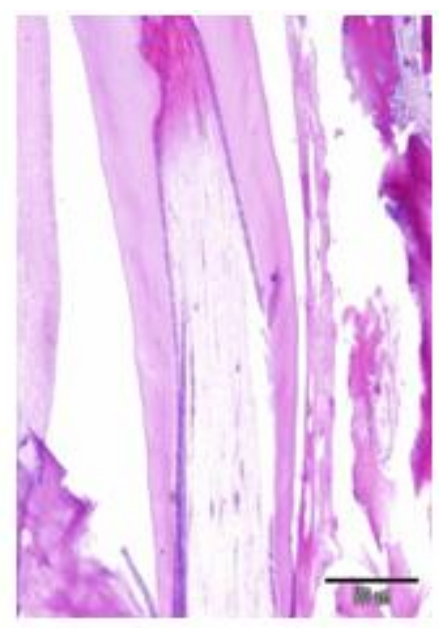

(a)

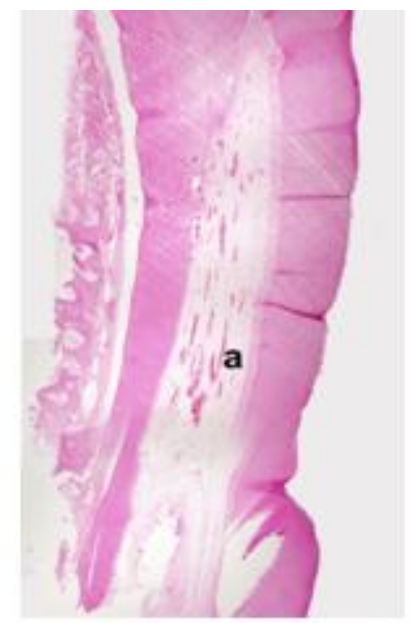

(b)

Fig 1: A photomicrograph of rabbit dental pulp after four weeks of application of capping materials:

a)

b) (a)

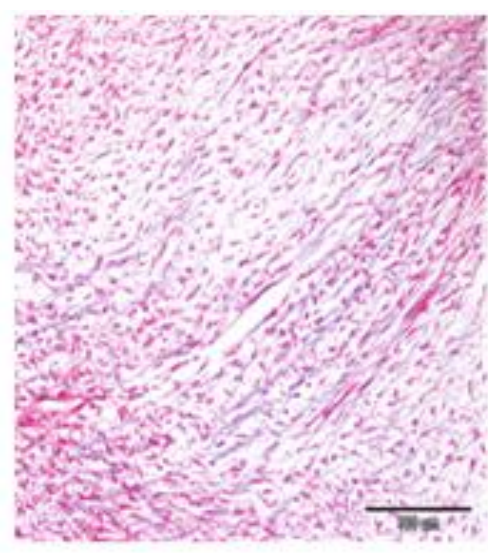

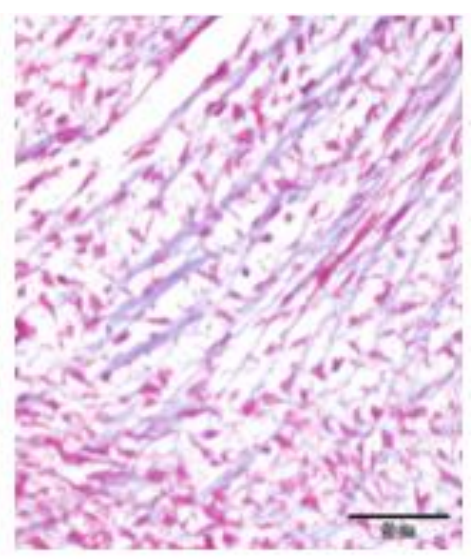

(b) a)

b)

Calcium hydroxide group: showing moderate dilatation of blood vessels in coronal and middle thirds

Fig 2: Photomicrograph of rabbit dental pulp after four weeks of application of capping materials: Propolis group: showing mild fibrosis.

Calcium hydroxide: showing moderate fibrosis. 


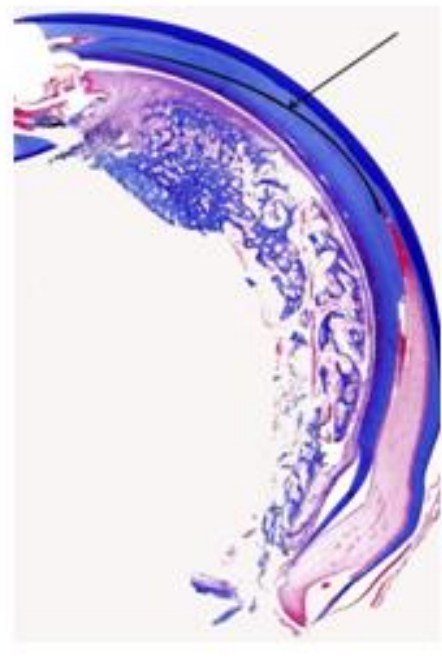

(a)

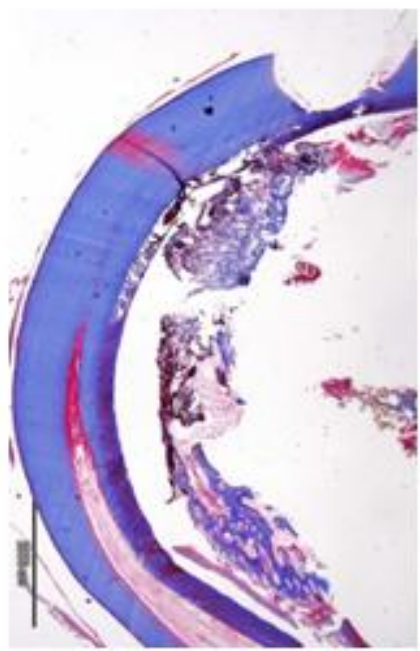

(b)

Fig3: Photomicrograph of rabbit dental pulp after four weeks of application of capping materials:

a) Propolis group: showing heavy hard tissue deposition beneath the capping material.

b) Calcium hydroxide group: showing moderate hard tissue deposition beneath the capping material.

\section{References:-}

1. Accornite ML, Loguerico AD, Reis A, Muench A, Araujo VC (2005): Response of human pulps capped with bonding agent after bleeding control with menostatic agents. Oper Dent;30: 147-155.

2. Ahangari Z, Naseri M, Jalili M, Mansouri Y, Mashhadiabbas F, Torkaman A (2012): Effect of propolis on dentin regeneration and the potential role of dental pulp stem cell in Guinea pigs. Cell J.;13(4):223-228.

3. Ansorge S, Reinhold D, Lendeckel U (2003): propolis and some of its constituents down-regulate DNA synthesis and inflammatory cytokine production but induce TGF-beta production of human immune cells. Z Naturforsch C. Jul-Aug; 58 (7-8) : 580- 589.

4. Belduz N, Yilmmaz Y, Ozbek E, Kalkan Y, Demirci T (2010). The effect of neodymium-doped yttrium aluminum garnet laser irradiation on rabbit dental pulp tissue. Photomed Laser Surg. Dec; 28 (6): 747-750.

5. Briso ALF, Rahal V, Mestrener SR, Dezan E Jr (2006): Biological response of pulps submitted to different capping materials. Braz Oral Res.; 20(3):219-225.

6. Costa CA, Giro EM, do Nascimento AB, Teixeira HM, Hebling J (2003): Short-term evaluation of the pulpo-dentin complex response to a resin-modified glass-ionomer cement and a bonding agent applied in deep cavities. Dent Mater. Dec;19(8):739-746.

7. Esmeraldo MR, Carvalho MG, Carvalho RA, Lime Rdef, CostaEM (2013): Inflammatory effect of green propolis on dental pulp in rats. Braz Oral Res. Sep- Oct: 27 (5) 417_422.

8. Estrela C, Pimenta FC, Ito IY, Bammann LL (1999): Antimicrobial evaluation of calcium hydroxide in infected dentinal tubules. J Endod, Jun; 25(6):416-418.

9. Fenwick N, Danielson P, Griffin G (2011): Survey of Canadian animal-based researchers' views on the Three Rs: replacement, reduction and refinement. PLoS One.; 6(8).

10. Fridland M, Rosado R (2005): MTA solubility: a long term study. J Endod. May;31(5):376-379.

11. Haddad M, Lefranc G, Aftimos G (2003): Local application of IGF1 on dental pulp mechanically exposed; in vivo study on rabbit. Bull Group Int Rech Sci Stomatol Odontol. Jan-Apr; 45(1): 12-17.

12. Khayyal MT, El-Ghazaly MA, El-Khatib AS (1993): Mechanism involved in the anti-inflammatory effect of propolis extracts. Drugs Exp Clin Res; 19: 197-203.

13. Konigswald Wv, Golenishev FN (1979): A method for determining growth rates in continuously growing teeth. Journal of Mammalogoy; 60: 397-400.

14. Koru O, Toksoy F, Acikel CH, Tunca YM, Baysallar M, Uskudar Guclu A, Akca E, Ozkok Tuylu A, Sorkun K, Tanyuksel M, Salih B. In vitro antimicrobial activity of propolis samples from different geographical origins against certain oral pathogens. Anaerobe. 2007 Jun-Aug;13(3-4):140-145. 
15. Luketić S, Malcić A, Jukić S, Anić I, Segović S, Kalenić S (2008): Coronal microleakage of two root-end filling materials using a polymicrobial marker. J Endod.;34(2):201-203.

16. Mustafa M, Saujanya KP, Jain D, Sajjanshetty S, Arun A, Uppin I, Kadri M (2012): Role of Calcium Hydroxide in Endodontics. Global Journal of Medicine and Public Health. 1(1):66-70.

17. Nascimento AB, FontanaUF, Teixeira HM, Costa CA (2000): Biocompatibility of a resin-modified glassionomer cement applied as pulp capping in human teeth. Am J Dent. Feb; 13 (1) : 28-34.

18. Ozorio JE, Carvalho LF, de Oliveria DA, de Sousa- Neto MD, Perez D (2012): Standardized propolis extract and calcium hydroxide as pulpotomy agents in primary pig teeth. J Dent Child (Chic). May- Aug; 79 (2): 53-58.

19. Park YK, Koo MH, Abreu JA, Ikegaki M, Cury JA, Rosalen PL (1998): Antimicrobial activity of propolis on oral microorganisms. Curr Microbiol. Jan;36(1):24-28.

20. Parolia A, Kundabala M, Rao NN, Acharya SR, Agrawal P, Mohan M, Thomas M (2010): A comparative histological analysis of human pulp following direct pulp capping with Propolis, mineral trioxide aggregate and Dycal. Aust Dent J. Mar; 55(1):59-64

21. Passcoe S, Gatehouse D (1986): The use of a simple haematoxylin and eosin staining procedure to demonstrate micronuclei within rodent bone marrow. Mutal Res. Aug; 164 (4): 273-243.

22. Sabir A, Abbu CR, Agustiono P, Sosroseno W (2005): Hisological analysis of rat dental pulp tissue capped with propolis. J Oral Sci. Sep; 47 (3): 135-138.

23. Scheller S, Ilewicz L, Luciak M, Skrobidurska D, Stojko A, Matuga W (1978): biological properties and clinical application of propolis. Experimental observation on the influence of ethanol extract of propolis (EEP) on dental pulp regeneration. Arzneimittelforschung.; 28 (2): 289-291.

24. Tziafas D, Smith AJ, Lesot H (2000): Designing new treatment strategies in vital pulp therapy. J Dent. Feb;28(2):77-92.

25. Woehrlen E (1997):. Evaluation of techniques and materials used in pulpal therapy based on a review of the literature: part 1. J Am Dent Assoc.; 97: 1154-1158.

26. Xu Y, Luo L, Chen B, Fu Y (2009): Recent development of chemical components in propolis. Frontiers of Biology in China.;4(4):385-391. 